\title{
X-ray Diffraction from the Ferroelectric Fluctuation and Domain Walls of Barium Titanate
}

\author{
Yasuhiro Yoneda, Yoshiki Kohmura ${ }^{1}$, Yoshio Suzuki ${ }^{2}$ \\ Synchrotron Radiation Research Unit, Japan Atomic Energy Agency (JAEA), \\ Kouto 1-1-1, Sayo-cho, Sayo-gun, Hyogo 679-5148 \\ Fax: 81-791-58-2740, e-mail: yoneda@spring8.or.jp \\ ${ }^{1}$ SPring-8/RIKEN, Kouto 1-1-1, Sayo-cho, Sayo-gun, Hyogo 679-5198 \\ ${ }^{2}$ SPring-8/JASRI, Kouto 1-1-1, Sayo-cho, Sayo-gun, Hyogo 679-5198
}

\begin{abstract}
A careful observation of X-ray diffraction in the ferroelectric crystals such as $\mathrm{BaTiO}_{3}$ reveals that a new physical picture of the ferroelectric transition in these crystals may rather be regarded as an order-disorder transition. A strong local order in the disordered phase is a characteristic feature. The model gives natural interpretation of the occurrence of a micro cluster phase in the phase transition to explain the existence of the depolarization phase, and this micro cluster phase existed just behind the phase transition temperature in the tetragonal phase.

Key words: $\mathrm{BaTiO}_{3}$, synchrotron X-ray topography, pair-distribution function
\end{abstract}

\section{INTRODUCTION}

Since the discovery of ferroelectricity in $\mathrm{BaTiO}_{3}$ in 1945 , it has been one of the most exhaustively studied materials [1]. At high temperatures, it has the classic $\mathrm{ABO}_{3}$ (with $\mathrm{Ba}^{2+}$ as $\mathrm{A}$ and $\mathrm{Ti}^{4+}$ as $\mathrm{B}$ ) perovskite structure. This is a centrosymmetric cubic structure with $\mathrm{A}$ at the corners, $\mathrm{B}$ at the center, and oxygens at the face centers. However, as the temperature is lowered, it goes through successive phase transitions to three different ferroelectric phases, each involving small distortions from the cubic symmetry. At $393 \mathrm{~K}$, it undergoes a paraelectric to ferroelectric transition to a tetragonal structure, it is orthorhombic between 278 and $183 \mathrm{~K}$ and, finally, it is rhombohedral below $183 \mathrm{~K}$. Each of these distortions can be thought of as elongations of the cubic unit cell along an edge ([001] or tetragonal), along a face diagonal ([011] or orthorhombic), or along a body diagonal ([111] or rhombohedral). These distortions result in a net displacement of the cations with respect to the oxygen octahedra along these directions. It is primarily these displacements that give rise to the spontaneous polarization in the ferroelectric phases.

Earlier theory by Mason and Matthias [2] assumed each titanium ion to be situated in a multiple-well potential field, and tried to interpret the ferroelectric transition as an order-disorder transition of the distribution of the Ti atoms among these potential wells. A subsequent paper by Devonshire [3], as well as one by Slater [4], denied the possibility of a multiple-well potential in the paraelectric phase, and gave a theory based on an single-well potential. The transition to ferroelectric phase occurs, just as in Mason-Matthias's theory, as a result of Lorentz interaction among Ti ions, but the transition is not of the order-disorder type but a simple simultaneous spontaneous shift of the $\mathrm{Ti}$ ions parallel to one crystallographic axis. Since then, the latter theory got wide acceptance and Mason-Matthias's theory seems to have been completely discarded. In 1961, Takahashi [5] reconsidered Mason-Matthias theory. Although it is doubtless that Mason and Matthias's assumption of a multiple-well potential for the $\mathrm{Ti}$ atoms is entirely inadequate for the paraelectric phase, the physical picture is strikingly akin to Devonshire and Slater's model, that is, the ferroelectric phase transition of barium titanate is properly an order-disorder transition.

In the experimental community, it is admitted that the purely displacive and order-disorder cases are merely limiting situations. The coexistence of displacive and order-disorder feature was also observed in $\mathrm{BaTiO}_{3}$. For example, central peak (CP), which is a signature of order-disorder case, and soft mode (SM), which is a signature of displacive case, are found to coexist by the spectroscopic studies. However, it is important in the material design to clarify the origin of the polarization. Originally, a displacive soft-mode concept has been used to explain the nature of the phase transitions of $\mathrm{BaTiO}_{3}$ $[6,7]$. On the other hand, the observation of diffuse X-ray scattering [8], persistent Raman spectra far above $T_{\mathrm{C}}[9,10]$, and the birefringence [11,12] suggested a formation of pretransition polar clusters in the paraelectric cubic phase. The microscopic nature of these polar precursors has recently been revealed by X-ray absorption fine structure [13] and nuclear magnetic resonance measurements [14] to be composed of dynamic off-center displacements of Ti ions along eight equivalent $<111>$ directions, consistent with the original eight-sight model suggested for the order-disorder component in $\mathrm{BaTiO}_{3}$. However, exact roles and microscopic natures of displacive and 
order-disorder components during the phase transition are still controversal.

The present experiments of the X-ray topography and $\mathrm{X}$-ray pair-distribution function (PDF) provide for the first time direct access to the static structure during the ferroelectric phase transition of $\mathrm{BaTiO}_{3}$, required for observation of $\mathrm{CP}$. The static structure that relates to $\mathrm{CP}$ has not been found yet. The analysis elucidates its relation to the order-disorder phase transition model. This paper thus settles the relation between the dynamical structure and the static structure, and suggests a path towards deeper understanding of structural phase transition in general.

\section{EXPERIMENTAL}

High-energy X-ray powder diffraction data were collected over a wide temperature range by using a closed-cycle helium refrigerator and an electric furnace with $60-\mathrm{keV}$ X-rays at BL04B2 and BL14B1 in SPring-8. The incident X-ray beam was focused on the samples by using a bent crystal $[15,16]$.

Samples of about $0.2 \mathrm{~g}$ were sealed between Kapton foils for the low-temperature data correction and were sealed in a quartz tube for the high-temperature data correction. Scattered radiation was collected with an intrinsic germanium detector. The data were corrected for background, absorption, multiple-scattering, and inelasticity effects and were normalized to the incident flux and the total sample scattering cross section to yield the total scattering structure function, $S(Q)$ [17]. This was Fourier transformed according to

$$
G(r)=\frac{2}{\pi} \int_{0}^{\infty} Q[S(Q)-1] \sin (Q r) d Q
$$

Structural information was extracted from the PDF by using trial structures to modeling in real space. The PDF of a model structure can be calculated by counting all the interatomic distances present in the crystal.

Both short-range and long-range structural parameters were refined by using the PDF method with the program PDFFIT [18] and by using a Rietveld refinement with the program RIETAN2000 [19], respectively.

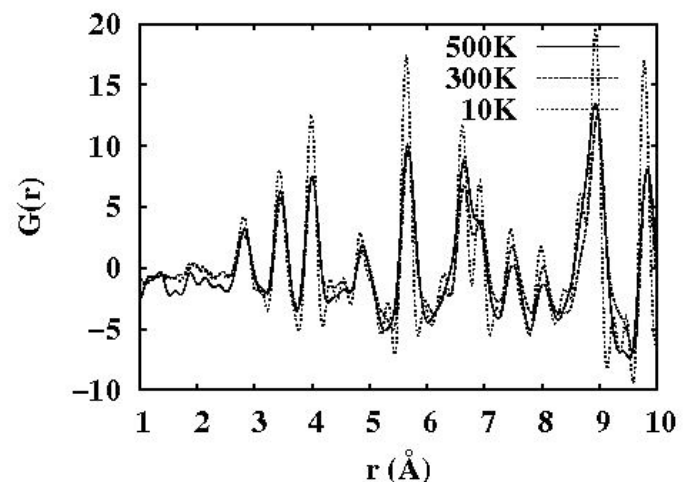

Fig. 1 Temperature evolution of the local structure of $\mathrm{BaTiO}_{3}$ from $10 \mathrm{~K}$ to $500 \mathrm{~K}$. The PDF spectra were unchanged at all measured temperatures, though ferroelectric phase transitions occurred at $190 \mathrm{~K}, 270 \mathrm{~K}$, and 400 K.
X-ray diffraction topography from a $\mathrm{BaTiO}_{3}$ crystal was studied in an X-ray diffractometer scheme at the optics beamline BL47XU, an undulator beamline of SPring-8. The undulator gap was $12 \mathrm{~mm}$ and $8 \mathrm{keV}$ $\mathrm{X}$-rays were used. X-ray energy was selected by a double Si (111) monochromator. Primary slit with vertical and horizontal gaps of $100 \times 100 \mu \mathrm{m}$ and $\phi=$ $20 \mu \mathrm{m}$ pinhole slit were used to improve the X-ray coherency. The diffractometer can achieve an angular resolution of $\sim 1$ arcsec. An ionization chamber counter was used to measure the diffracted intensity. When the $\mathrm{X}$-ray diffraction topography performed, the reflected beam image was recorded by a 2D X-ray detector (Beam Monitor, BM) with the pixel size of $40 \mu \mathrm{m}$. The BM consisted of a fluorescent screen and a CCD (C4742, Hamamatsu Photonics Co.). A $\mathrm{BaTiO}_{3}$ single crystal was grown by the top-seeded solution growth method, which was provided from MTI Co. in USA. The crystal was vergin sample in a sense with no external electric field applied prior to the measurements. It was cut in a cube with $5 \mathrm{~mm}$ with the thickness of $1.5 \mathrm{~mm}$. The sample was supported in a small cupper cell of the milli-Kelvin (mK)-stabilized cell [20] and the sample temperature was controlled within $\pm 1 \mathrm{mK}$.

\section{RESULTS}

3.1 X-ray PDF analysis

We identified the local structure of $\mathrm{BaTiO}_{3}$ by using the pair-distribution function (PDF) method [21]. Since the PDF is determined from both the Bragg and the diffuse scattering intensities, information on short-range correlated atom displacements has been obtained Figure 1 provides the temperature evolution of the local structure of $\mathrm{BaTiO}_{3}$. The peak positions and peak shapes do not change at any measured temperature. Although $\mathrm{BaTiO}_{3}$ has three phase transitions in the measured temperature range and the average structure changed, the local structure remained unchanged. The local bond distribution clearly shows that the phase transition of

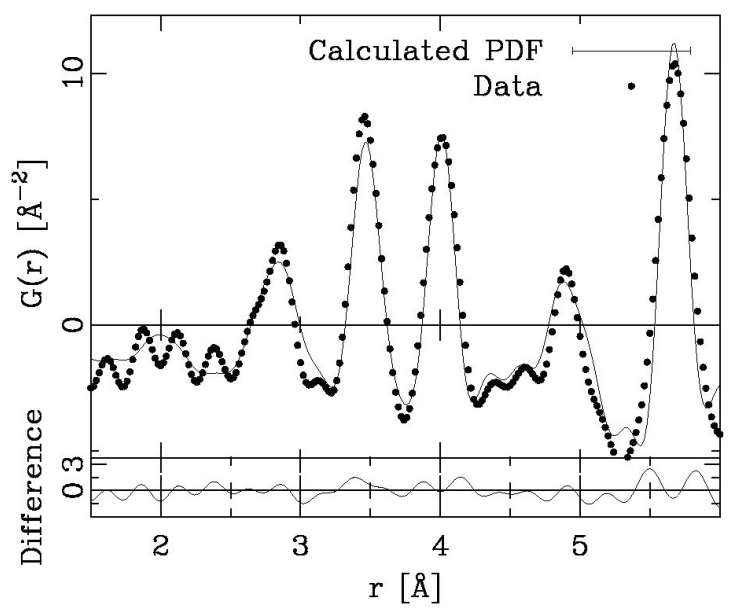

Fig. 2 Comparison between the experimental PDF (closed circles) and the calculated PDF (solid line) for $\mathrm{BaTiO}_{3}$ at $200 \mathrm{~K}$ (cubic phase). Difference curves are plotted below the data. 
$\mathrm{BaTiO}_{3}$ is not the displacive case but the order-disorder case. We tried to fit the experimental PDFs to clarify the difference in the local structures of $\mathrm{BaTiO}_{3}$ at the cubic phase. Since the local structure of $\mathrm{BaTiO}_{3}$ did not change at any measured temperature, we adopted the same convention as used for the rhombohedral average structure to calculate the PDF for $\mathrm{BaTiO}_{3}$ in the cubic phase. When the fitting range was $1.5<r<6.5 \AA$, there was a somewhat large deviation between the calculated and the observed PDFs. When the fitting range was 1.5 $<r<6.0 \AA$, the agreement was clearly very good, as revealed in Fig. 2. The local structure analysis also clarify that the dynamic off-center displacements of the rhombohedral structure remains in the paraelectric cubic phase. Different local structure models are required, depending on the fitting range. The so-called "box-car" refinement [22,23] should be adopted for the local structure analysis of $\mathrm{BaTiO}_{3}$. Note that there seems to be some ghost peaks around $r=2 \AA$ owing to the Fourier termination error. The Fourier termination error of an $\mathrm{X}$-ray PDF tends to be remarkable compared to that of the neutron PDF. The nearest neighbor Ti-O peak is one peak in reality, as shown by the calculated PDF in Fig. 2.

\subsection{X-ray diffraction topography}

The temperature dependence of the crystal coherence at the domain boundary was visualized in the beam profile [24,25]. When the crystal net plane tilted owing to the lattice strain and domain fluctuation, the reflected beams scattered, depending on the tilt angle of the net plane. On the other hand, when the X-rays hit an unstrained area, a sharp spot was observed. The net plane is tilted around the domain boundary. Even if the temperature is precisely controlled, the fine structure of the (002) Bragg reflection that is divided in the line shape due to the distortion from the domain boundary is observed.

Figure 3 provides the temperature dependence of the beam size. The beam size was shown by the pixel size of the CCD camera. The lattice fluctuation around the domain boundary reflects the bending mode of the surface in the $\mathrm{BaTiO}_{3}$ stripe domain. This bending mode

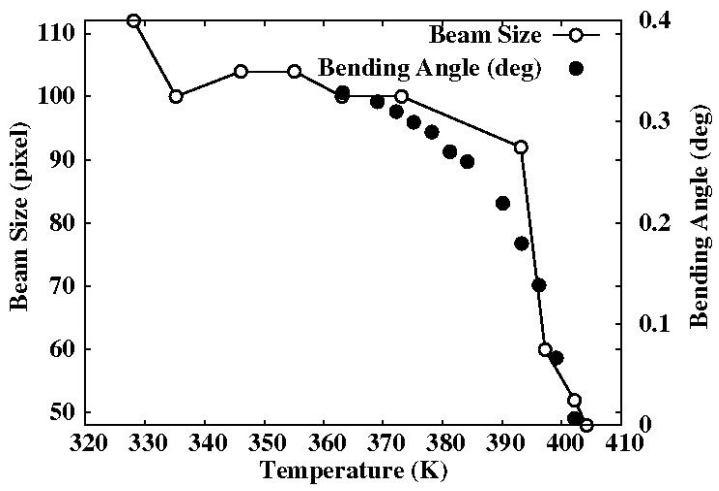

Fig. 3 Temperature dependence of beam size shown in pixel size of CCD camera. The data agree well with the surface bending angle obtained by the AFM technique. has been also investigated by Takashige et al. using atomic force spectroscopy (AFM) [26]. Takashige's data correspond well with our data. The surface bending mode coupled with the local strain at the domain boundary. The local strain at the domain boundary reduced as temperature increased. Finally, the uniform strain covered the entire domain just before the phase transition occurred. The role of the local strain at the domain boundary is to stabilize the ferroelectric domain. The reduction of the local strain at the domain boundary means the domain becomes unstable.

The preparation for changing the domain configuration was complete. The ferroelectric domain did a complex change in a very narrow temperature range. Figure 4(a) provides the CCD image of the X-ray topography of the $\mathrm{BaTiO}_{3}$ just before the phase transition temperature of $402.4 \mathrm{~K}$. Since the local strain reduced, the spot size from the tetragonal domain was very small. The diffraction pattern changed greatly immediately after that. The diffraction spot extended suddenly, as revealed in Fig. 4(b). The domain size of the unstable tetragonal phase became very small right under the phase transition [25]. The small micro domain configuration existed only in a very little temperature range, and the reflection of the high temperature cubic phase appeared at once. Figure 4(c) provides the CCD image at $404.5 \mathrm{~K}$ where the tetragonal and the cubic reflections were coexistent. These were diffuse pattern because either domain was small and unstable. The domain in the phase transition neighborhood of the $\mathrm{BaTiO}_{3}$ did a drastic change in the temperature range of only $0.1 \mathrm{~K}$.

\section{DISCUSSION}

We have found a clear evidence of the order-disorder phase transition of $\mathrm{BaTiO}_{3}$, which is unchanged local bond distribution through three phase transitions. The rhombohedral local structure of the off-center displacements along eight equivalent $\langle 111\rangle$ directions remained in the high-temperature cubic phase. The microscopic nature of order-disorder components was clarified by the static local structure of the PDF. Our structure analysis is corresponding to the results of neutron PDF [27,28].

We also reexamined the phase transition of the $\mathrm{BaTiO}_{3}$ by using the X-ray diffraction topography. As a result, it has been understood that the tetragonal - cubic phase transition occurs by three stages. At the first stage, the lattice strain at the domain boundary uniformly
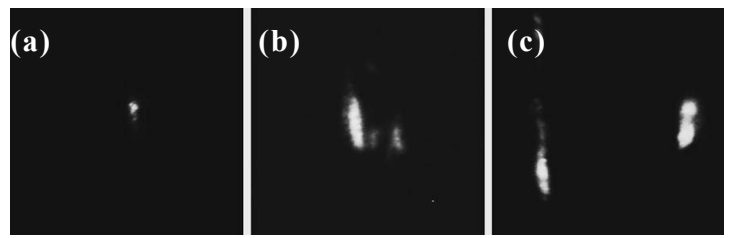

Fig. 4 CCD images of X-ray diffraction topography of $\mathrm{BaTiO}_{3}$ single crystal during tetragonal-cubic structural phase transition. The measurement was performed during heating. 
extends to the entire domain. Since the ferroelectric domain is stabilized by the strain localized at the domain boundary, the domain configuration of the tetragonal phase became unstable. Then, the ferroelectric domain changed very small at the second stage. The polarization was free from the local strain, that is, the depolarization occurred. The depolarization temperature, which has been reported in $\mathrm{Bi}_{0.5} \mathrm{Na}_{0.5} \mathrm{TiO}_{3}$ [29] and $\mathrm{BiFeO}_{3}-\mathrm{BaTiO}_{3}$ mixture [30] etc., also existed in the pure $\mathrm{BaTiO}_{3}$ in a very little temperature range. And finally, the average structure changed from tetragonal to cubic structure in the third stage. Because the order-disorder phase transition does not change the local structure, such a complex domain change becomes possible.

It has been believed that at least near the vicinity of $T_{\mathrm{C}}$, the ferroelectric phase transition shows an order-disorder nature, owning to the observation of CP. The CP involves interwell motion by Ti ions parallel to the polar axis. On the other hand, the $\mathrm{CP}$ is possible to interpret traveling cluster waves [31,32]. The CP phenomenon is due to the lifetime of the cluster waves. Close to $T=T_{\mathrm{C}}$ and close to the displacive limit, these traveling cluster wave give rise to a cluster excitation branch appearing in addition to the phonon resonances. Our result of the X-ray topography supports the cluster wave, because we observed the microdomain, which will cause the anharmonicity of thermal motions. Though there is room for argument on this point, the possibility to relate the depolarization phenomena and the $\mathrm{CP}$ could be suggested.

\section{SUMMARY}

We performed X-ray PDF and X-ray topography of $\mathrm{BaTiO}_{3}$. The local structure unchanged in wide temperature range. This behavior strongly suggests the order-disorder phase transition. The fine structure of the Bargg reflection revealed that the domain size became very small just before the phase transition temperature. The micro cluster that caused the depolarization was able to observe even in the tetragonal phase.

\section{ACKNOWLEDGMENTS}

The synchrotron radiation experiments were performed at the BL47XU in the SPring-8 with the approval of the Japan Synchrotron Radiation Research Institute (JASRI) (Proposal No. 2006A1110). This work was supported by a Grant-in-Aid for Young Scientists (No. 19740186) from the Ministry of Education, Culture, Sports, Science and Technology.

\section{REFERENCES}

[1] G. Shirane, F. Jona and R. Pepinsky, R. Proc. IRE, 43, 1738 (1955) : F. Jona and G. Shirane, Ferroelectric Crystals, Pergamon Press, New York (1962).

[2] W. P. Mason and B. T. Mattias: Phys. Rev. 74, 1622 (1948).

[3] A. F. Devonshire: Phil. Mag. (7) 40, 1040 (1944).

[4] J. C. Slater, Phys. Rev. 78, 748 (1950).

[5] H. Takahashi: J. Phys. Soc. Jpn. 16, 1685 (1961).

[6] W. Cochran: Adv. Phys. 9, 387 (1960).

[7] J. Harada, J. D. Axe, and G. Shirane: Phys. Rev. B 4,
155 (1971).

[8] R. Comes, M. Lambert, and F. Gervais: J. Phys. C 13, 3761 (1980).

[9] S. Wada, T. Suzuki, M. Osada, M. Kamihara, and T. Noma: Jpn. J. Appl. Phys., Part1 37, 5385 (1998).

[10] U. M. Pasha, H. Zheng, O. P. Thakur, A. Feteira, K. R. Whittle, D. C. Sinclair, and I. M. Reaney: Appl. Phys. Lett. 91, 062908 (2007).

[11] M. Takagi and T, Ishidate: Solid State Commun. 113, 423 (2000).

[12] A. Ziebinska, D. Rytz, K. Szot, M. Grorny, and K. Roleder: J. Phys.: Condens. Matter 20, 142202 (2008).

[13] B. Ravel, E. A. Stern, R. I. Vedrinskii, and V. Kraisman, Ferroelectrics 206, 407 (1998).

[14] B. Zalar, V. V. Laguta, and R. Blinc: Phys. Rev. Lett. 90, 037601 (2003).

[15] Y. Yoneda, N. Matsumoto, Y. Furukawa and T. Ishikawa: J. Synchrotron Rad. 8, 18 (2001).

[16] Y. Yoneda, N. Matsumoto, Y. Furukawa and T. Ishikawa: Nuclear Instruments and Method in Physics Research A 467-468, 370 (2001).

[17] X. Qiu, J. W. Thompson, and S. J. L. Billinge: $J$. Appl. Cryst. 37, 678 (2004).

[18] Th. Proffen and S. J. Billinge: J. Appl. Crystallogr. 32, 572 (1999).

[19] F. Izumi and T. Ikeda: Mater. Sci. Forum 321-324, 198 (2000).

[20] A. Kojima, Y. Yoshimura, H. Iwasaki, K. Tozaki, Its Measuremenmt and Control in Science and Technology, vol.7, part 1, Springer, New York 2003, p. 921.

[21] T. Egami and S. J. L. Billinge: Underneath the Bragg Peaks: Structural Analysis of Complex Materials (Elsevier Science, Oxford, 2001).

[22] K. Kodama, S. Iikubo, S. Shamoto, A. A. Belik, and E. Takayama-Muromachi: J. Phys. Soc. Jpn. 76, 124605 (2007).

[23] X. Qiu, E. S. Bozin, P. Juhas, Th. Proffen, and S. J. L. Billinge: J. Appl. Crystallogr. 37, 110 (2004).

[24] Y. Yoneda, Y. Kohmura, Y. Suzuki, S. Hamazaki and M. Takashige, J. of Phys. Soc. of Jpn. 73, 1050 (2004).

[25] Y. Yoneda, J. Mizuki, Y. Kohmura, Y. Suzuki, S. Hamazaki and M. Takashige, Jpn. J. of Appl. Phys. 43, 6821 (2004).

[26] M. Takashige, S. Hamazaki, Y. Takahashi, F. Shimizu and T. Yamaguchi, Jpn. J. Appl. Phys. 38, 5686 (1999).

[27] G. H. Kwei, A. C. Lawson, S. J. L. Billinge and S. -W. Cheong, J. Phys. Chem. 97, 2368 (1993).

[28] G. H. Kwei, S. J. L. Billinge, S. -W. Cheong, and J. G. Saxton, Ferroelectrics 164, 57 (1995).

[29] N. Yasuda, S. Hashimoto, H. Ohwa, O. Sakurada, K. Fujita, Y. Yamashita, M. Iwata, and Y. Ishibashi, Jpn. J. Appl. Phys. 48, 09KC06 (2009).

[30] Y. Yoneda, K. Yoshii, S. Kohara, and S. Mori, Jpn. J. Appl. Phys. 47, 7590 (2008).

[31] T. Schneider and E. Stoll: Phys. Rev. Lett. 35, 296 (1975).

[32] T. Schneider and E. Stoll: Phys. Rev. B 13, 1216 (1976).

(Received December 25, 2009; Accepted January 31, 2010) 